\title{
Initial Experiments in the Cooperative Control of Unmanned Air Vehicles
}

Derek R. Nelson

Brigham Young University - Provo

Timothy W. McLain

Brigham Young University - Provo, mclain@byu.edu

Reed S. Christiansen

Aero Vironment, Inc.

Randal W. Beard

Brigham Young University - Provo, beard@byu.edu

David Johansen

Brigham Young University - Provo

Follow this and additional works at: https://scholarsarchive.byu.edu/facpub

Part of the Mechanical Engineering Commons

\section{BYU ScholarsArchive Citation}

Nelson, Derek R.; McLain, Timothy W.; Christiansen, Reed S.; Beard, Randal W.; and Johansen, David, "Initial Experiments in the Cooperative Control of Unmanned Air Vehicles" (2004). Faculty Publications. 1525.

https://scholarsarchive.byu.edu/facpub/1525

This Peer-Reviewed Article is brought to you for free and open access by BYU ScholarsArchive. It has been accepted for inclusion in Faculty Publications by an authorized administrator of BYU ScholarsArchive. For more information, please contact ellen_amatangelo@byu.edu. 


\title{
Initial Experiments in Cooperative Control of Unmanned Air Vehicles
}

\author{
Derek R. Nelson ${ }^{1},{ }^{*}$ Timothy W. McLain ${ }^{1}$, Reed S. Christiansen ${ }^{2}$, \\ Randal W. Beard ${ }^{1}$, David Johansen ${ }^{1}$ \\ ${ }^{1}$ Brigham Young University Provo, UT 84602 \\ ${ }^{2}$ AeroVironment, Inc. Monrovia, CA 91016
}

\begin{abstract}
This paper addresses cooperative control for a team of unmanned air vehicles (UAVs). Specifically, a team of three small UAVs is controlled to perform a cooperative timing mission. Starting at loiter locations distributed around the periphery of a $2 \mathrm{~km}$ square battle area, the UAVs cooperatively plan paths to arrive at a target at the center of the battle area in sequence at $10 \mathrm{sec}$ intervals. Cooperative path planning is performed using the methodology of coordination variables and coordination functions. Coordination and waypoint path planning are centralized on a ground station computer. Experiments have been performed using BYU's fleet of small fixed-wing UAVs, which employ BYU's autopilot and ground station technology. Experimental results demonstrating cooperative timing are presented.
\end{abstract}

\section{Introduction}

Unmanned air vehicles (UAVs) are playing increasingly prominent roles in the nation's defense programs and strategy. While drones have been employed in military applications for many years, the dramatic growth in technological capabilities in recent years has increased their utility and versatility. Specifically, advances in microcontrollers, sensors, and batteries have enabled both the significant improvements seen in large UAVs (e.g., Global Hawk, Predator), and the creation of smaller, increasingly capable UAVs. The focus of our efforts at BYU has been on these smaller miniature UAVs (MUAVs), which range in size from $1 \mathrm{ft}$ to $6 \mathrm{ft}$ in wingspan. There are numerous military applications for mini-UAVs including reconnaissance, surveillance, battle damage assessment, and communication relays. These applications are of current and pressing interest and significant resources are being directed towards their development. ${ }^{1}$

Civil and commercial applications are not as well developed, although the potential applications are extremely broad in scope. Possible applications for MUAV technology include environmental monitoring (e.g., pollution, weather, scientific applications), forest fire monitoring, homeland security, border patrol, drug interdiction, aerial surveillance and mapping, traffic monitoring, precision agriculture, disaster relief, ad-hoc communications networks, and rural search and rescue. For many of these applications to develop to maturity, reliability of MUAVs will need to increase, their capabilities will need to be extended further, their ease of use will need to be improved, and their cost will have to come down.

Spurred by recent increased interest in UAVs from the military community, research activity in the area of cooperative control of UAV systems has been high. Progress has been made in theoretical areas such as cooperative search ${ }^{2-4}$ cooperative path planning, ${ }^{5}$ and cooperative control strategies. ${ }^{6,7}$ Experimental work with teams of UAVs has been limited, primarily due to the practical challenges of fielding multiple vehicles simultaneously. At the present, most UAV cooperative control experiments have focused on demonstrations of leader following with two UAVs. ${ }^{8-10}$ The work presented here shows the execution of a cooperative timing mission by a team of three UAVs. A unique characteristic of this experimental work is that the cooperation occurs in path planning and assignment rather than in trajectory tracking.

\footnotetext{
${ }^{*}$ Corresponding author, email:mclain@byu.edu
} 


\section{Problem Overview}

This paper addresses a cooperative surveillance problem where a team of UAVs is directed to fly over a target with a specified time interval between the time over target for each vehicle. An example scenario of this cooperative mission is depicted in Figure 1. During the initial phase of the mission, the UAVs perform preprogrammed tasks independent of each other. These tasks could be search or surveillance operations, or as in the work presented here, a simple loiter task. On command from the operator on the ground, cooperative path plans are computed for the UAVs. Based on the location of the UAVs at the time the command is issued, these paths lead the UAVs over the target at the specified time spacing and the same heading. In this way, a persistent image of the target can be acquired. The paths selected minimize the battery energy required to complete the fly by of the target. Upon completion of the fly by, the UAVs return to their independent tasks until the next cooperative fly-by command is received. The primary challenge in this scenario is the real-time demands placed on the cooperative path planning.
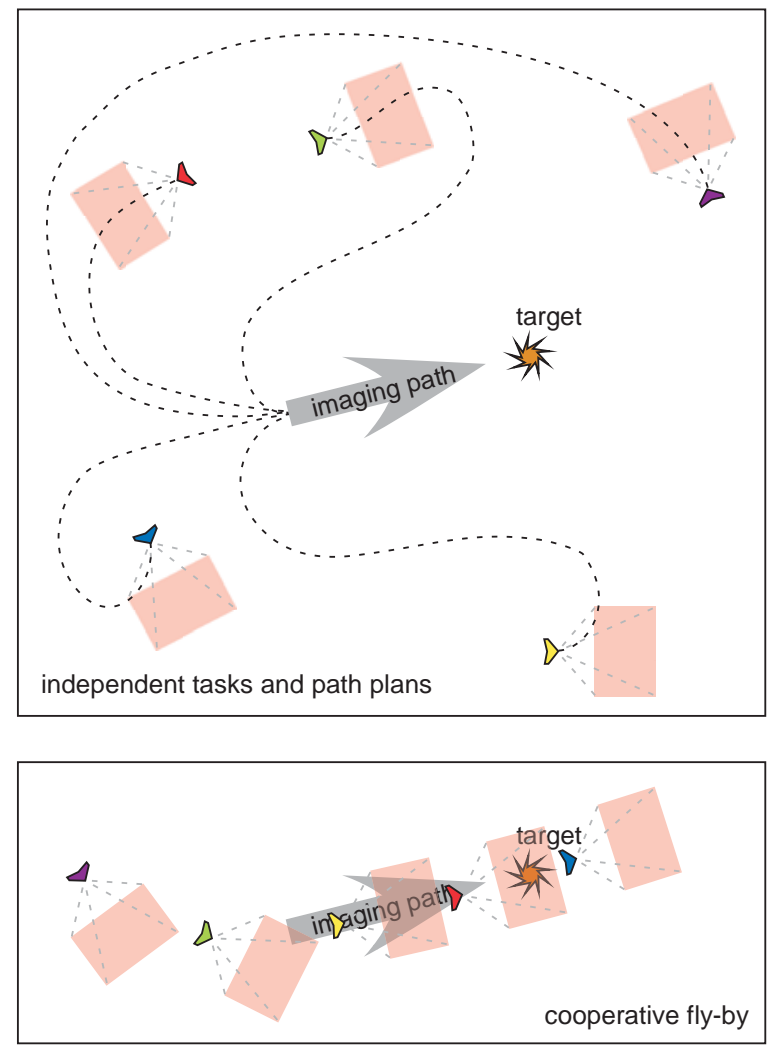

Figure 1. Cooperative fly-by scenario.

Once a cooperative fly-by command is issued, candidate path plans are issued for each of the UAVs. These paths are based on the current location of the UAV, the target location, and the desired fly-by heading. Candidate waypoint paths are calculated that satisfy the mission specifications. The arrival order of the UAVs, their velocities, and the specific waypoints they fly are selected by optimization based on coordination function information for each vehicle. Coordination variables and coordination functions have proven useful for cooperative control problems such as the fly-by scenario addressed here ${ }^{11}$ and will be briefly discussed.

\section{Technical Approach}

The underlying concept of coordination variables and coordination functions is that the minimal information essential to achieving a cooperation objective should be identified and communicated among vehicles on the team. Let $\mathbf{x}_{i}$ define the situation state for the $i^{\text {th }}$ vehicle on a cooperative team. For the cooperation 
problem considered here, the situation state includes information about a UAV's current position, the target position, the fly-by heading, and the time interval between UAV arrivals. Additional information about the environment (e.g., wind speed and direction) can also be included. For a given situation $\mathbf{x}_{i}$, the set of feasible decisions for a vehicle is given by $\mathcal{U}_{i}\left(\mathbf{x}_{i}\right)$ and $\mathbf{u}_{i} \in \mathcal{U}_{i}$ is the decision variable for the $i^{\text {th }}$ vehicle. The choice of the decision variable by each vehicle on the team affects both the feasibility and the quality of the cooperation achieved. In the fly-by scenario, the decision variable vector is consists of path waypoint and velocity information.

The process of cooperation among agents can be viewed as having objectives and constraints. For the fly-by scenario, the cooperation constraint requires the UAVs to fly over the target with the second vehicle at a specified time interval behind the first, and the third vehicle arriving at a specified time interval after the second. Cooperation is said to occur if the cooperation constraints are met.

The quality of the cooperation is quantified by the cooperation objective. In the fly-by scenario, the cooperation objective is to minimize the battery energy required to complete the mission. The contribution of a vehicle to the team cooperation objective is represented by an influence function, $\phi_{i}=J_{i}\left(\mathbf{u}_{i}\right)$. In this case the energy consumption for the $i^{\text {th }}$ vehicle (represented by $\phi_{i}$ ) is a function of the UAV velocity and the waypoints flown (represented by $\mathbf{u}$ ). The coordination variable $\theta$ represents the minimal amount information necessary to achieve cooperation. For the fly-by problem, the coordination variable is the arrival time of the first UAV and the arrival order for the vehicles. If every vehicle knows the value of the coordination variable and responds accordingly, cooperative behavior will be achieved by the team. For a vehicle, the value of the coordination variable is related to the decision variable, $\theta_{i}=f_{i}\left(\mathbf{u}_{i}\right)$, and defines what the vehicle can do to ensure that cooperation constraints are met. In the fly-by scenario, the UAVs choice of path and velocity determines its energy consumption and arrival time and therefore influences the cooperation objective and the cooperation constraint.

Under the assumptions that battery power is proportional to aerodynamic drag and that the UAV flies at constant speed, the battery energy consumption to fly to the target is given by

$$
J_{i}=c_{b} v_{i} L_{i}
$$

where $c_{b}>0$ is a constant, $v_{i}$ is the UAV velocity, and $L_{i}$ is the length of the waypoint path taken.

For the cooperative fly-by scenario with three UAVs, the cooperation constraint can be written as

$$
\begin{aligned}
& T_{1}=T_{s} \\
& T_{2}=T_{s}+\Delta_{2} \\
& T_{3}=T_{s}+\Delta_{3}
\end{aligned}
$$

where $T_{i}$ is the arrival time of the $i^{\text {th }}$ vehicle, $\Delta_{i}$ represents the interval between the arrival of the first and $i^{\text {th }}$ vehicles, and $\theta=T_{s}$ is the coordination variable.

Critical to the implementation this approach is the definition of the coordination function. The coordination function models an vehicle's influence on the cooperation objective in terms of what the agent can do to meet the cooperation constraints. The coordination function, $\phi_{i}\left(\theta_{i}\right)$ is derived from the influence function and coordination variable definitions as

$$
\begin{aligned}
\phi_{i} & =J_{i}\left(\mathbf{u}_{i}\right) \\
& =J_{i}\left[f_{i}^{\dagger}\left(\theta_{i}\right)\right] \\
& =\phi_{i}\left(\theta_{i}\right),
\end{aligned}
$$

where $f_{i}^{\dagger}$ is the pseudoinverse of $f_{i}$. Typically, $f_{i}$ is not a one-to-one mapping in that numerous values of the decision variable (waypoint paths, velocities) can result in a single value of the coordination variable (time over target). The pseudoinverse of $f_{i}$ is found by taking the value of decision variable that minimizes the influence function $J_{i}$, thus creating a one-to-one map between $\theta_{i}$ and $\mathbf{u}$. In other words, when multiple options exist to get the same result, the lowest-cost option is chosen.

Using coordination variables and coordination functions, the battery energy required complete the mission can be minimized by solving the optimization problem

$$
\theta^{*}=\arg \min _{\theta=T_{s}}\left[\phi_{1}\left(T_{1}\right)+\phi_{2}\left(T_{2}\right)+\phi_{3}\left(T_{3}\right)\right] .
$$


subject to the constraint of Equation (1).

Once a team optimal value for the coordination variable is found, vehicle decisions can be found from the relationship

$$
\mathbf{u}_{i}=f_{i}^{\dagger}\left(\theta^{*}\right) .
$$

\section{Experimental Setup}

A reliable and robust platform for multi-agent UAV testing has been developed, starting with the BYU MAGICC Lab's Kestrel autopilot, shown in Figure 2. The autopilot is equipped with a Rabbit $300029 \mathrm{MHz}$ processor, rate gyros, accelerometers, as well as absolute and differential pressure sensors. The autopilot measures only 1.5 by 2 by $3 / 4$ inches and weighs 18 grams. The airframe chosen was the Zagi XS EPP foam flying wing (Figure 3). This platform was chosen for its durability, desirable flying characteristics, and ease of installation of additional components needed for autonomous flight, including the radio modem, GPS unit, video transmitter, and autopilot. BYU has also developed Virtual Cockpit software for programming and controlling UAV flight, along with a ground station transmitter with the capability of handling multiple aircraft, shown in Figure 4. The aircraft and ground station transmitter use Aerocomm $44901000 \mathrm{~mW}$, $900 \mathrm{MHz}$ wireless modems for communications. The aircraft are also equipped with a Furuno GH-80D GPS unit.

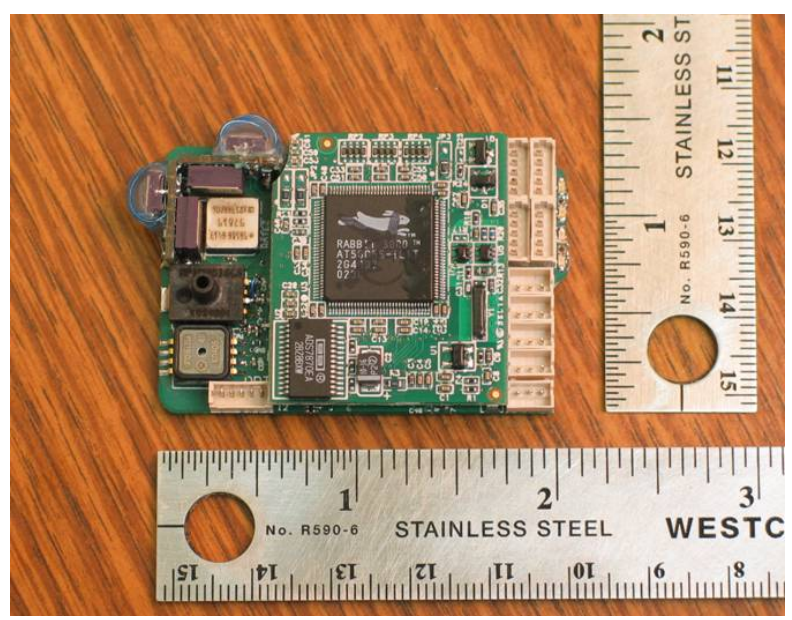

Figure 2. Kestrel autopilot.

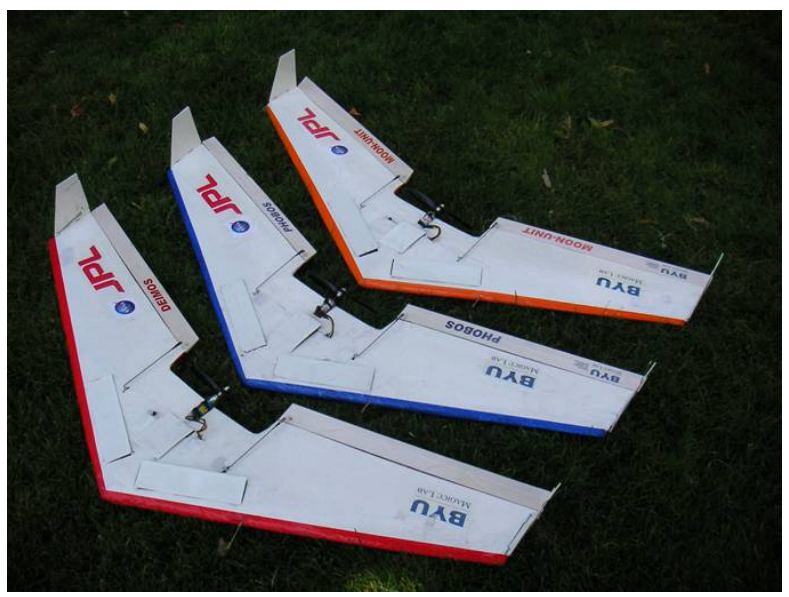

Figure 3. Zagi airframe. 


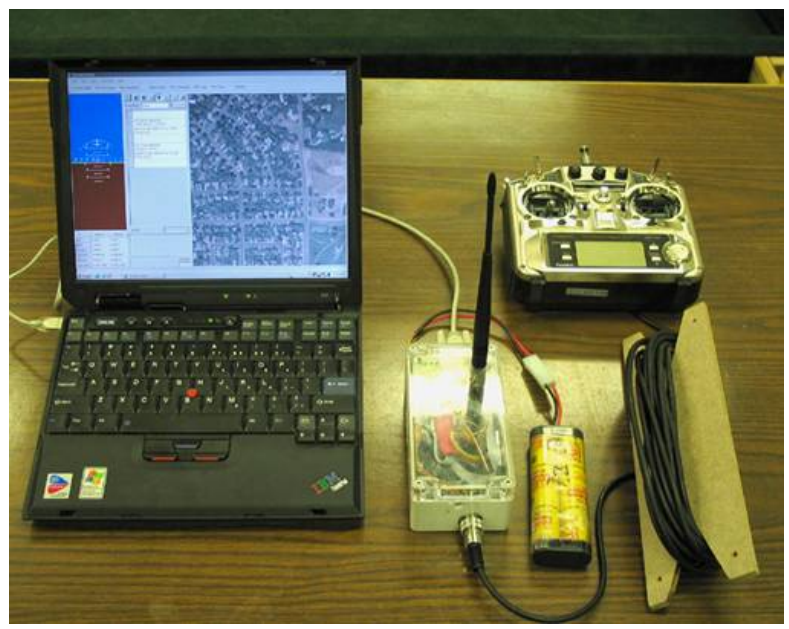

Figure 4. Virtual Cockpit and ground station.

For this initial implementation of the cooperative fly-by problem, the path planning requirements for the individual UAVs were straightforward. First, there were no threats or obstacles to be avoided. Second, the target location and fly-by heading were specified. For each UAV, a single path composed of straight-line segments from the UAV's current location to the target on the desired heading was adequate to meet the mission requirements.

Figure 5 shows an example of the waypoint paths used in the experiment. The first waypoint for each agent was calculated to be 50 meters directly ahead of the initial position of the UAV. The second waypoint was defined to be a fixed distance ahead of the target location along the desired fly-by heading. The next waypoint was the target location. The fourth waypoint was collinear with waypoints 2 and 3 and was the same distance opposite the target as was point 2. Points 2, 3, and 4 were the same for each agent and were referred to as fly-through points. The fifth waypoint was included to help the agents distance themselves from the target before returning to their final loiter positions, point 6 .

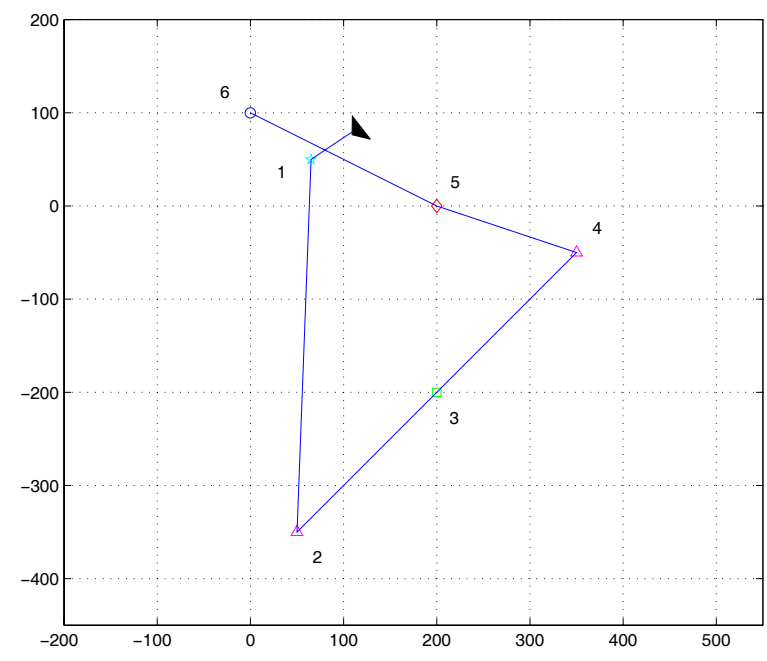

Figure 5. Waypoint path example.

The cooperative timing algorithm was implemented on the ground station computer. Once the UAVs were in the air and performing their individual tasks, the operator executed the cooperative fly-by algorithm. The algorithm used for this work, was carried out in the following steps: 
1. Acquire GPS locations and headings for each UAV.

2. Calculate waypoint paths for each UAV.

3. Compute range of arrival times for each UAV.

4. Compute coordination function for each UAV.

5. Based on coordination functions, choose team optimal coordination variable (arrival time of first UAV, arrival order).

6. Calculate desired velocity for each UAV.

7. Send waypoint paths and desired velocities to each UAV.

Figure 6 illustrates the coordination functions calculated for a cooperative fly-by based on input information provided to the algorithm. Because the coordination function for each UAV is monotonically decreasing, the team-optimal arrival time for one of the vehicles will always lie at the right extreme of its coordination function. Determining the minimum energy arrival time and order for the team involves a simple search through the right extreme (minimum) values of each coordination function with an evaluation of the team objective to determine the best values.

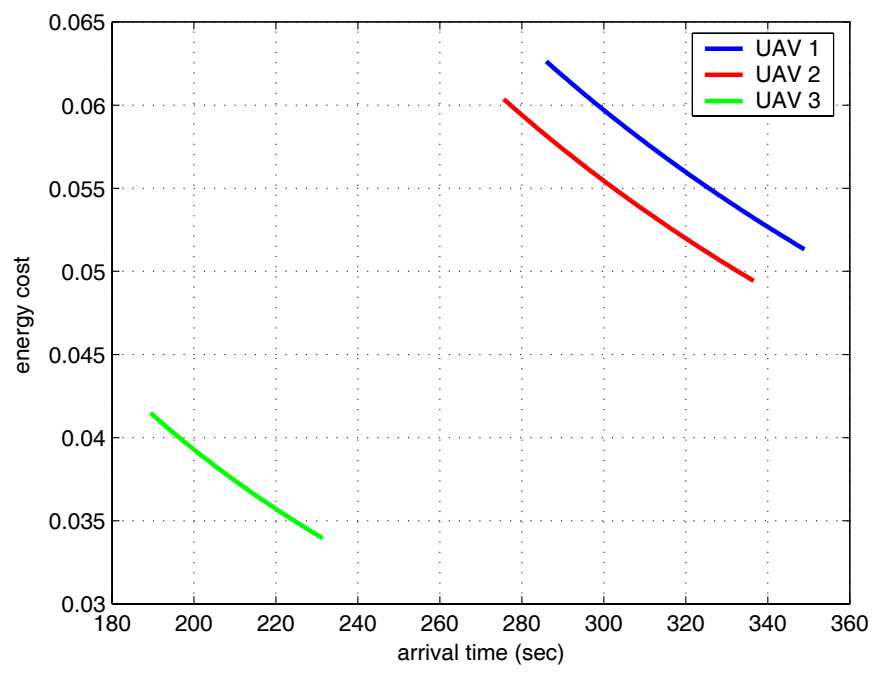

Figure 6. Initial coordination functions.

In some cases, depending on the initial UAV positions and headings, the coordination functions do not yield a solution that satisfies the cooperative timing constraint. For the problem considered in Figure 6, a time spacing of 10 seconds was required. Based on the coordination functions calculated from the waypoint paths, it can be easily seen that this was not feasible. In these instances, it became necessary to develop some simple heuristics for adding length to one or more paths so that the timing constraints could be met.

Based on the coordination functions, the length to be added to a path to ensure constraint feasibility was calculated. If the length that needed to be added was less than 50 meters, the location of the first waypoint was adjusted using the law of cosines so that the total distance would be the original length plus the necessary additional length. If the additional length was longer than 50 meters, two additional waypoints were added to the path immediately following the first waypoint. The second waypoint was calculated so that its distance from the first waypoint was half of the required additional distance, and the waypoint was placed in a direction directly opposite the target. The third waypoint was placed at the same location as the first, thus effectively adding the required length to the path.

Figure 7 shows how the coordination function for UAV 3 was modified by adding length to the original path. With the added length, the coordination function shifted up and to the right allowing the 10 second interval timing constraint to be satisfied. 


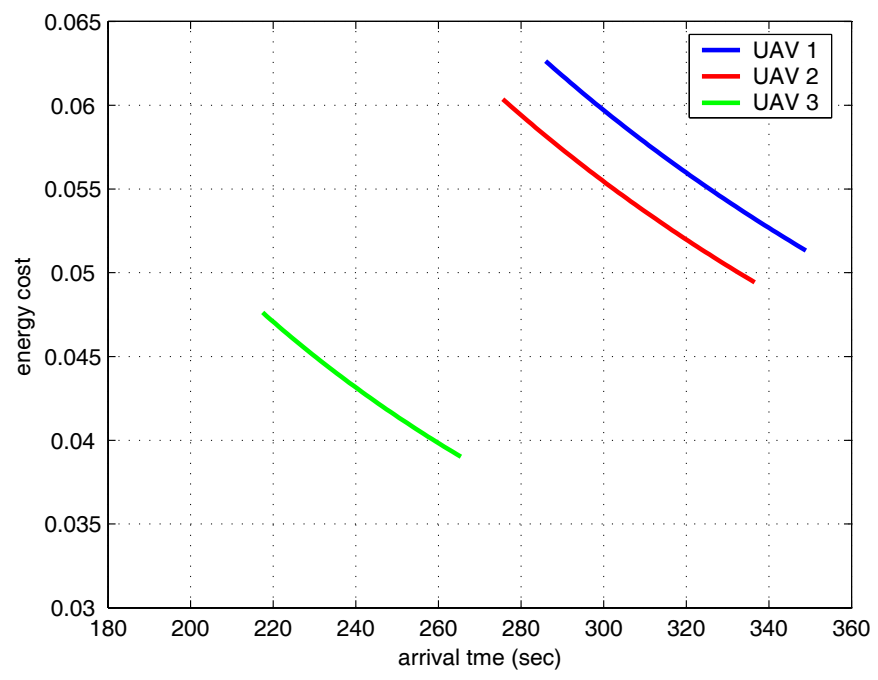

Figure 7. Final coordination functions.

The cooperative fly-by algorithm was implemented as part of the Virtual Cockpit ground station software. The implementation was a centralized solution, in that the ground station acquired all the information required to do the cooperative timing calculations from the vehicles, performed the calculations and sent only the paths and desired velocities back to be flown by the UAVs. Execution of the algorithm took approximately one second, with a significant portion of the time utilized for communication between the UAVs and the ground station. Once the UAVs received the paths and velocities, there was no further feedback to ensure proper timing. The onboard autopilot controlled each vehicle by maintaining a constant airspeed and adjusting the heading so that the vehicle was directed towards the next waypoint.

\section{Results and Discussion}

For the test results presented here, three UAVs were put into the air and commanded to loiter at specified locations. The UAVs were then commanded to perform a cooperative fly-by and return to their loiter points. A second fly-by command was given after which the UAVs returned again to their loiter points. This implementation did not correct for trajectory tracking errors due to wind disturbances, the inability of the vehicles to track sharp corners in the waypoint paths, or airspeed sensing inaccuracies. The weather conditions had a significant impact on the results of the test. On the day of the fly-by test, the winds were 6 to $8 \mathrm{~m} / \mathrm{sec}$ or approximately 30 to 50 percent of the UAV airspeed. The desired interval for arrival for the vehicles at the target was 10 seconds between the first and second vehicle and the second and third vehicle. The first of the three-vehicle fly-by passes had actual arrival intervals of 25 and 6 seconds, while the second fly-by pass had intervals of 10 and 9 seconds.

Snapshots showing an overhead view of the second fly-by pass, plotted from the telemetry data, can be seen in Figure 8. Initially the UAVs were loitering around the locations marked with circles. The coordination algorithm was called and the UAVs flew to their first waypoint (not plotted), followed by the fly-through waypoints. The magenta triangles indicate the fly through entry and exit points and the green square marks the target that the vehicles coordinated around. Window (b) of Figure 8 shows the UAVs en route to the entry waypoint. Windows (c), (d), and (e) show the arrival of the first, second, and third UAVs at the target. After the fly by, the vehicles returned to their loiter position as shown by window (f).

The cause of the large timing interval error in the first fly-by was due to the high wind speeds present during the test. Both UAV 2 and UAV 3 were headed upwind while UAV 1 was flying downwind when the cooperative fly-by algorithm was executed. UAVs 2 and 3 took significantly longer to reach their first waypoint than did UAV 1 . Since the target was downwind from the loiter positions, UAV 1 had a large head start. This accounts for the 25 second interval between UAV 1 and UAV 2. The 6 second interval between UAVs 2 and 3 is reasonable due to the wind conditions. The second fly-by yielded better results because the 

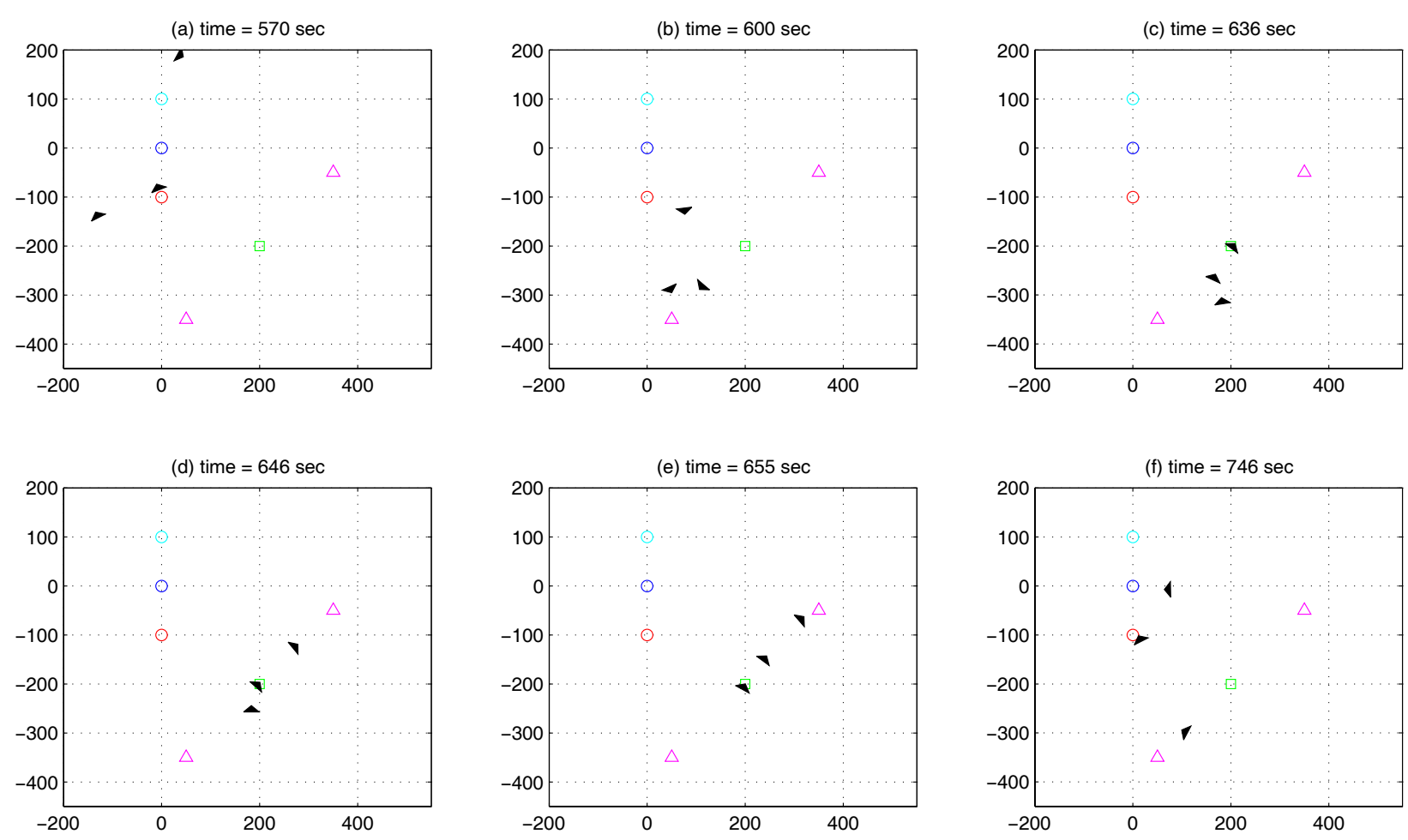

Figure 8. Cooperative fly-by results.

cooperative fly-by algorithm was executed when all three vehicles were flying in a crosswind direction. With all of the vehicles in a similar wind condition initially, the intervals between arrival times were much closer to what was desired.

Although these initial results are promising, there are several improvements that could be made to improve the robustness and practical feasibility of the approach. Implementation of a trajectory generator and tracker would make the UAVs more robust to wind disturbances. Currently, the cooperative timing is based on ground speed, while the control of the UAVs is based on air speed. When wind speeds are significant, this can result in timing errors. Periodic updates of the range to target could be used to adjust UAV velocities while the mission is underway. This too would increase robustness to tracking and ground speed errors. While the current centralized implementation yields excellent results, improved scalability for larger numbers of vehicles could be realized by decentralizing more of the computations to the individual vehicles.

\section{Conclusions}

The experimental results presented here demonstrate that real-time cooperative control with miniature UAVs is possible and can be done effectively. Three UAVs were flown simultaneously using a single ground station to coordinate their efforts. Cooperative fly-by missions were successfully flown using an algorithm based on coordination functions and variables. Although preliminary, the results are promising and demonstrate the feasibility of the approach.

\section{Acknowledgments}

This work was funded by AFOSR grant FA9550-04-1-0209. 


\section{References}

1 "Unmanned Aerial Vehicle Roadmap 2002-2007," Tech. rep., Office of the Secretary, U.S. Department of Defense, December 2002

${ }^{2}$ Beard, R. and McLain, T., "Multiple UAV Cooperative Search Under Collision Avoidance and Limited Range Communication Constraints," Proceedings of the IEEE Conference on Decision and Control, December 2003, pp. 25-30.

${ }^{3}$ Jin, Y., Minai, A., and Polycarpou, M., "Cooperative Real-Time Search and Task Allocation in UAV Teams," Proceedings of the IEEE Conference on Decision and Control, December 2003, pp. 7-12.

${ }^{4}$ Yang, Y., Minai, A., and Polycarpou, M., "Decentralized Cooperative Search by Networked UAVs in an Uncertain Environment," Proceedings of the American Control Conference, July 2004, pp. 5558-5563.

${ }^{5}$ Bellingham, J., Tillerson, M., Alighanbari, M., and How, J., "Cooperative Path Planning for Multiple UAVs in Dynamic and Uncertain Environments," Proceedings of the IEEE Conference on Decision and Control, December 2002, pp. $2816-2822$.

${ }^{6}$ Inalhan, G., Stipanovic, D., and Tomlin, C., "Decentralized Optimization with Application to Multiple Aircraft Coordination," Proceedings of the IEEE Conference on Decision and Control, December 2002, pp. 1147-1155.

${ }^{7}$ Tiwari, A., Fung, J., Carson, J., Bhattacharya, R., and Murray, R., "A Framework for Lyapunov Certificates for MultiVehicle Rendezvous Problems," Proceedings of the American Control Conference, July 2004, pp. 5582-5587.

${ }^{8}$ Seanor, B., Campa, G., Gu, Y., Napolitano, M., Rowe, L., and Perhinschi, M., "Formation Flight Test Results for UAV Research Aircraft Models," AIAA 1st Intelligent Systems Technical Conference, September 2004, AIAA Paper No. $2004-6251$.

${ }^{9}$ King, E., Kuwata, Y., Alighangari, M., Bertucelli, L., and How, J., "Coordination and Control Experiments on a MultiVehicle Testbed," Proceedings of the American Control Conference, July 2004, pp. 5315-5320.

${ }^{10}$ Campbell, M., D'Andrea, R., Lee, J., and Scholte, E., "Experimental Demonstrations of Semi-Autonomous Control," Proceedings of the American Control Conference, July 2004, pp. 5338-5343.

${ }^{11}$ McLain, T. W. and Beard, R. W., "Coordination Variables, Coordination Functions, and Cooperative Timing Missions," AIAA Journal of Guidance, Control and Dynamics, 2004, (to appear). 